\title{
Aplicaciones de las nanotecnologías en los estudios sobre el agua: un tema de interés estratégico para Iberoamérica y España
}

\author{
Pedro A. Serena*
}

En los países más desarrollados el acceso al agua es tan común, y por lo general a un coste tan relativamente bajo, que sus habitantes no se plantean la importancia que tiene este líquido elemento en todo nuestro desarrollo. De la misma forma, no se es consciente del papel clave que el agua ha tenido y tiene para el desarrollo de la vida en la Tierra. Con este argumento seguramente sobran los demás para saber que nos encontramos frente a un tema de capital importancia.

Como no podía ser de otra forma, el desarrollo de la humanidad ha estado ligado a la capacidad de ingeniar y utilizar tecnologías vinculadas al agua: tecnologías de extracción, acumulación, distribución, eliminación y depuración de aguas residuales, sistemas optimizados de riego, sistemas de transporte fluviales y marítimos, contención de aguas, desalación de agua marina, obtención de alimentos mediante piscicultura, obtención de energía eléctrica en saltos hidráulicos, etc. ${ }^{1} \mathrm{~A}$ lo largo de la historia, el desarrollo de las civilizaciones ha caminado de forma paralela a una creciente demanda de recursos hídricos. Sin embargo, la utilización de éstos ha terminado concentrándose en una pequeña parte de la humanidad (UNDP, 2006). Otro claro ejemplo de reparto desigual de los resultados del progreso humano. En un extremo, países como Estados Unidos y Australia tienen un consumo promedio, respectivamente, de 575 y 500 litros diarios por habitante, cantidad que en los países europeos se reduce a 200-350 litros por habitante y día. En el entorno iberoamericano, podemos mencionar que el consumo de agua en México se encuentra en los 365 litros diarios por habitante, y en España es de unos 320 litros por habitante y día. Ambos países se encuentran en la franja del consumo alto de agua. Por el contrario hay un gran número de regiones en los que el consumo está por debajo de unas pocas decenas de litros por día, incluso por debajo de los límites que aseguran la supervivencia.

Es obvio constatar que el consumo de agua en un país va ligado al factor combinado de la industrialización del mismo y de su ubicación en zonas climáticas secas que requieren grandes cantidades para usos agrícolas y ganaderos. Únicamente mil millones de personas tienen un acceso fiable al agua potable, precisamente aquellas que viven en países desarrollados. Otros cuatro mil millones de habitantes tienen problemas cotidianos para acceder a este recurso, y unos mil millones viven en condiciones de claro "déficit hídrico". Como consecuencia de esta dificultad para conseguir agua

\footnotetext{
* Instituto de Ciencia de Materiales de Madrid, Consejo Superior de Investigaciones Científica, c/ Sor Juana Inés de la Cruz, 3, 28049-Cantoblanco, Madrid, e-mail: pedro.serena@icmm.csic.es.

1 La relación entre el desarrollo de la humanidad y las tecnologías del agua queda reflejada en la serie de artículos aparecidos durante 2006 y 2007 en la revista Tecnología del Agua (editada por la empresa Reed Bussiness Information) dentro de la sección "Agua y Culturas" (http://www.rbi.es/nuevo/index.html).
} 
potable, cinco millones de personas enferman gravemente cada año, llegando a fallecer dos millones de niños por esta causa (UNDP, 2006). En cuanto al acceso al agua, los países iberoamericanos no tienen problemas excesivos, pues Haití es el único país que garantiza el acceso potencial (agua sin tratar) al líquido elemento a menos del 70\% de su población. Sin embargo, la situación empeora si nos referimos al agua tratada o potabilizada, pues países como Venezuela, Guadalupe, Perú, El Salvador, Bolivia, y Haití no garantizan el $70 \%$ de cobertura (UNDP, 2006).

Aunque el consumo de agua está ligado claramente al grado de desarrollo industrial de un país no podemos olvidar que el continuo aumento de la población del planeta obliga al crecimiento global de la producción agrícola y ganadera, ejerciéndose así una gran presión sobre los recursos hídricos. Se estima, por ejemplo, que la cantidad de agua necesaria para producir $1 \mathrm{~kg}$ de cereales es de 1,500 litros de agua, y que $1 \mathrm{~kg}$ de carne de vacuno representa un gasto de 15,000 litros de agua (Hoekstra y Hung, 2002). Este tipo de estimaciones realizadas para diferentes tipos de alimentos o de productos procesados ha permitido establecer los conceptos de "agua virtual" y de "huella hídrica", definiendo mapas de flujo de agua virtual entre los países (Hoekstra y Hung, 2002; Chapagain et al., 2005).

En paralelo al consumo básico de agua, el mantenimiento o aumento de unas cotas de bienestar en los países desarrollados se traduce en un aumento imparable del consumo de agua. Decir, como ejemplo, que en el mundo se consumen 148,000 millones de litros de agua envasada al año. Otro ejemplo bastante llamativo de la relación entre "progreso" y consumo hídrico lo podemos encontrar en un país como España, que cuenta con cerca de 600,000 piscinas. $^{2}$ Si tenemos en cuenta este número, su capacidad media, y la necesidad de renovar cada día un $5 \%$ del volumen almacenado, encontramos que cada día se necesitan casi seis mil millones de litros diarios únicamente para renovar el agua de las piscinas españolas. Si tenemos en cuenta que, según datos del Foro Mundial del Agua, ${ }^{3}$ un ser humano necesita para sobrevivir alrededor de 50 litros al día, el agua consumida en España para el mantenimiento de las piscinas podría servir para garantizar al acceso a agua tratada de 120 millones de personas cada día. Este dato se puede comparar con los más de mil millones de personas en todo el mundo que no tienen acceso a esta cantidad mínima de agua apta para el consumo. Como consuelo, podemos mencionar que la mayor parte de las piscinas españolas únicamente están en funcionamiento en los meses de verano.

Esta creciente demanda ha conducido a una explotación abusiva de manantiales, aguas de superficie y aguas subterráneas. Las primeras consecuencias de estas actuaciones son de índole ecológica, pues determinan cambios irreversibles de los ecosistemas, o deterioros en los acuíferos, como la contaminación de éstos por nitratos y fosfatos, o por arsénico. Sin embargo, no hay que olvidar las consecuencias sociopolíticas, ya que la presión por los escasos recursos hídricos genera tensiones entre países (o entre regiones dentro de un país). Estas tensiones se vislumbran como posible origen de enfrentamientos armados a corto y medio plazo (Watkins y Berntell, 2006; Schwartz y Singh, 1999). Las Naciones Unidas se han marcado una serie de retos dentro del Proyecto Milenio (Millenium Proyect, 2005a, 2005b y 2005c) y entre ellos destaca el objetivo de lograr que el $25 \%$ de la población mundial tenga un acce-

\footnotetext{
2 http://revista.consumer.es/web/es/20030601/actualidad/informe1/.

3 Consejo Mundial del Agua: http://www.worldwatercouncil.org/.
} 
so razonable al agua apta para el consumo. Esta iniciativa contribuirá al alivio temporal de parte de los focos de tensión internacional, retrasando el estallido de conflictos mientras se establecen soluciones de carácter más definitivo.

En este contexto turbulento, a lo largo de la última década han aparecido una serie de informes (IPCC, 2007; Al Gore, 2007) que confirman la existencia de un rápido -en términos geológicos- e inevitable cambio climático, alertando sobre sus consecuencias: el deshielo de los polos (representando una gran pérdida de agua dulce), el avance acelerado de los desiertos, las pérdidas de selvas y humedales, la desecación de lagos en zonas templadas, modificaciones en el régimen de lluvias, entre otros. Es decir, a la situación de escasez actual de recursos, que se deben compartir entre una población mundial que crece de manera imparable, debemos añadir un nuevo ingrediente perturbador: el cambio climático. Los países iberoamericanos están grandemente afectados por las consecuencias del cambio climático, al encontrarse ubicados en zonas secas o selváticas, que pueden sufrir más rápidamente la expansión de los desiertos o el deterioro de las masas forestales, junto con la intensificación de fenómenos como "El Niño" o el número de huracanes en las regiones caribeñas.

Ante este panorama tan preocupante, tan sólo queda replantear de forma global el modelo de desarrollo en el que hemos asentado nuestro progreso desde la llegada de la revolución industrial y dejar paso a nuevos esquemas como el planteado por desarrollo sostenible (WCED, 1987), el cual plantea actuaciones en muchos frentes (energía, vivienda, transporte, etc). Uno de los aspectos fundamentales del modelo del desarrollo sostenible es la necesidad de alcanzar una mejor, más racional y más eficiente utilización de los recursos hídricos.

Esta nueva forma de plantear el diseño de nuestro desarrollo debe involucrar todas las perspectivas: la mejora y optimización de infraestructuras, la educación de la ciudadanía, la cooperación internacional, la valoración de la generación/pérdida de agua en términos de impacto medioambiental, por mencionar algunos. Además, y de forma obligada, se deben incentivar las actividades de investigación y desarrollo sobre el agua (desalación, descontaminación, depuración, detección y medición de contaminantes, eficiencia de su transporte y gestión, etc.), actividades que deben abordarse desde una perspectiva multidisciplinar, pues este tipo de enfoques es el que puede producir avances más rupturistas.

España, Perú, Bolivia, Chile y México son unos de los principales actores sobre este escenario de la escasez de recursos, dada su ubicación geográfica en las fronteras de la imparable desertización, y dada la enorme necesidad de recursos hídricos que se requieren para asegurar el progreso de estos países (en el esquema actual de desarrollo insostenible). En España, como se indicó, el consumo de agua por persona es de unos 320 litros/día lo que nos sitúa entre los diez países de mayor consumo. Un 80\% de este consumo se dedica a regadío, un $14 \%$ al abastecimiento de núcleos urbanos y un $6 \%$ a usos industriales. ${ }^{4}$ La combinación de estos niveles de consumo y nuestro modesto régimen de lluvias nos convierten en el cuarto país del mundo en número de grandes presas (unas 1,200). También, como ya se ha mencionado anteriormente, países como México no van a la zaga en cuanto a consumo de agua, y en el Distrito Federal se llegan a consumir del orden de 400 litros por habitante y día.

\footnotetext{
4 Información sobre la situación del agua en España se encuentra en la página web http://www.miliarium. com/Monografias/, que incluye informes sobre el Plan Hidrológico Nacional y el Programa AGUA (Actuaciones para la Gestión y la Utilización del Agua).
} 
En el caso de España, el consumo de agua es elevado, tal y como corresponde a un país desarrollado con una poderosa agricultura, favorecida por un clima benigno, pero que, en muchos casos, sigue usando sistemas ineficientes de riego. Este esquema se puede aplicar a otros países como México, y en menor medida a Chile o Perú. La sobrexplotación de los acuíferos y malas prácticas agrarias han hecho que muchos de estos acuíferos se contaminen por nitratos y fosfatos. En España, muy recientemente, se han dado casos de contaminación por arsénico de varios acuíferos, afectando a decenas de miles de personas (como la contaminación del acuífero de Los Arenales en Castilla-León (García-Sánchez et al., 2005; Martín-Gil et al., 2002)). Esta misma forma de contaminación se está dando de forma masiva en Bangla Desh, pero la situación en Chile, Argentina o México no se puede catalogar de optimista (Smith et al., 2000). Por citar un ejemplo, más de dos millones de habitantes de los estados mexicanos de Chihuahua, Coahuila, Durango, Hidalgo, Nuevo León y Puebla, se encuentran expuestos a beber agua con niveles de arsénico que ponen en riesgo su salud. ${ }^{5}$

Además de la presión agrícola, países como España y México reciben decenas de millones de turistas (UNTWO, 2008) deseosos de poder disfrutar de alimentos sanos, agua para el aseo, piscinas, etc. Más aún, la necesidad de aumentar la "calidad" de la oferta turística hace necesaria la promoción de nuevos elementos de atracción, como ocurre con los parques acuáticos o los campos de golf. En relación con esta práctica deportiva, España cuenta en la actualidad con casi 350 campos de golf, visitados por más de medio millón de turistas extranjeros. Paradójicamente, el 35\% de estos campos están ubicados en las comunidades autónomas de Andalucía y Murcia, regiones con más de la mitad de su territorio bajo la amenaza de desertización. ${ }^{6}$ Éste es otro ejemplo real de las situaciones a las que lleva un progreso económico carente de todo criterio de sostenibilidad.

Esta forma de plantear el crecimiento económico, la alarma por el cambio climático, la mayor información a la que los ciudadanos pueden acceder, entre otros, han favorecido la creciente preocupación social por los temas relacionados con el agua. Como ejemplo de este debate social podemos citar parte de los hechos ocurridos en España en esta última década: las movilizaciones a favor y en contra de los transvases desde la cuenca del río Tajo a la del Segura, la encrespada discusión política sobre las paralizadas obras del transvase del Ebro contempladas en el Plan Hidrológico Nacional, 7 la incorporación en los nuevos Estatutos de Autonomía de las Competencias sobre la Gestión de las Aguas de Ríos, o el debate sobre la construcción de una veintena de plantas desaladoras dentro del Programa AGUA lanzado por el Ministerio de Medioambiente ${ }^{8}$. Seguramente se pueden encontrar analogías con toda esta problemática en otros países como México, Chile, Bolivia o Perú. No cabe duda de que la gestión del agua es un problema estratégico para estos países y que el agua, como ele-

\footnotetext{
5 Noticia aparecida en La Jornada, México DF, 23 de junio de 2006, haciéndose eco de la celebración en México del Congreso Internacional As 2006 (Arsénico Natural en Aguas Subterráneas de América Latina).

6 Informe del Programa de Acción Nacional contra la Desertificación, del Ministerio de Medio Ambiente de España. Informe accesible en http://www.mma.es/portal/secciones/biodiversidad/desertificacion/.

7 La información sobre el Programa AGUA del Ministerio de Medio Ambiente se encuentra en http://www. mma.es/secciones/agua/entrada.htm.

$8 \mathrm{Ibid}$. Información sobre la situación del agua en España se encuentra en la página web http://www.miliarium.com/Monografias/, que incluye informes sobre el Plan Hidrológico Nacional y el Programa AGUA (Actuaciones para la Gestión y la Utilización del Agua).
} 
mento estratégico, debería ser una de las prioridades de las actividades de I+D de estos países.

La nanotecnología es un área multidisciplinar que amalgama conocimientos científico-técnicos provenientes de diversas áreas y, por lo tanto, posee áreas de aplicación muy extensas. Así, es lógico pensar en la existencia de puntos de encuentro entre la nanotecnología y el estudio del agua, tal y como ponen de manifiesto diferentes informes norteamericanos ${ }^{9}$ y europeos (Saxl, 2005). Por lo general, en estos informes el tema del agua no tiene la misma importancia en comparación con otros temas de mayor peso para los sectores industriales de los países más desarrollados (electrónica, informática, telecomunicaciones, automoción, aeronáutica, biomedicina, defensa, etc.). Dichos informes incluyen, de forma vaga, algunas líneas de investigación relacionadas con las tecnologías del agua: diseño y fabricación de nanosensores, técnicas analíticas para medir la calidad del agua; técnicas de filtración y purificación basadas en la nanotecnología usando sistemas de membranas fabricadas con materiales multifuncionales, desarrollo de productos textiles hidrófobos que no se ensucien fácilmente, producción de vegetales de bajo consumo de agua, etc. Si nos fijamos en lo ocurrido en España, la Acción Estratégica de Nanociencias y nanotecnologías del anterior Plan Nacional de I+D+i 2004-2007 ${ }^{10}$ no incluye líneas donde se produzca de forma clara el encuentro entre la nanotecnología y los diversos aspectos relacionados con el agua. Sin embargo, esta situación se ha corregido de forma parcial en el Plan Nacional de I+D+i 2008-2011, ${ }^{11}$ que incluye una Acción Estratégica de Nanotecnología con una línea de actuación en temas medioambientales (que puede incluir parte de la temática "agua").

Esta situación de débil interacción entre los temas agua y nanotecnología se ha ido corrigiendo en los últimos años como se demuestra con diferentes evidencias. Por un lado, se han celebrado conferencias internacionales en las que la conjunción de ambos temas ha sido la protagonista. ${ }^{12}$ Por otro, han aparecido varios informes internacionales en los que se demuestra el amplio abanico de aplicaciones de la nanotecnología en el ámbito del agua (Meridian Institute, 2005, 2006a, 2006b; Salamanca-Buentello, 2005). Además, se puede constatar que las actuaciones en este ámbito tienen como potenciales beneficiarios a los miles de millones de habitantes que viven en países pobres o en vías de desarrollo, por lo que la nanotecnología es una herramienta clave, según las Naciones Unidas, para alcanzar algunos de los objetivos del Proyecto Milenio (Millenium Proyect, 2005a, 2005b, 2005c).

Siguiendo los informes de la Fundación Meridian (Meridian Institute, 2005, 2006a, 2006b) las aplicaciones de la nanotecnología en el ámbito de los estudios sobre el agua se pueden clasificar de acuerdo con estas temáticas:

- Membranas para nanofiltración (Ng y Ong, 2006). Desarrollo de nanomateriales (basados en nanotubos de carbono (Srivastava et al., 2004), sistemas porosos

\footnotetext{
9 "The National Nanotechnology Initiative. Strategic Plan" (2004); véase The National Nanotechnology Initiative; Research and Development Leading to a Revolution in Technology and Industry, Supplement to President's FY 2007 Budget" (2006). Estos documentos se pueden encontrar en http://www.nano.gov.

${ }^{10} \mathrm{http}: / /$ www.mec.es/ciencia/plan_idi/.

${ }_{11} \mathrm{http} / / /$ www.plannacionalidi.es/plan-idi-public/documentos/plan_nacional_08-11.pdf.

${ }^{12}$ Nanowater Conference 2004, http://www.cientifica.eu/. Commercialising Nanotechnology in Water 2006. Melbourne, Australia. International Workshop on Nanotechnology, Water \& Development, 10-12 de octubre de 2006, Chennai, India. 3rd Annual Greener Nanoscience Conference 2008 (Corvallis, Oregon, EUA).
} 
de alumina, por mencionar algunos) para construir estructuras con dimensiones, densidades y formas controladas, capaces de filtrar selectivamente virus, bacterias, etcétera.

- Filtros basados en nanopolímeros, arcillas de atapulgita y zeolitas. Estos elementos naturales ya han sido usados como filtros desde hace tiempo pero la nanotecnología permite modificar algunas de sus propiedades (como el tamaño de poro o la inclusión de nanopartículas bactericidas) para hacerlos más eficientes (Prashant y Pradeep, 2005).

- Mejora de técnicas de desalación (ósmosis inversa) mediante nanotecnología. El proceso de osmosis inversa (Wilf et al., 2007), usado en las plantas desaladoras convencionales, puede mejorar su eficiencia mediante la adecuada combinación de nanomembranas y sistemas de nanofiltrado.

- Nanopartículas (Tiwari et al., 2008). Las nanopartículas de dióxido de titanio, de hierro, plata, zinc, etc., se pueden usar como catalizadores para degradar contaminantes orgánicos y eliminar sales y metales pesados de los líquidos en los que están disueltos. En el caso de las nanopartículas magnéticas, es posible recubrirlas con diferentes componentes con afinidad selectiva por ciertas sustancias contaminantes. Mediante la aplicación de campos magnéticos es posible también la eliminación de las sustancias que se adsorban sobre la nanopartícula magnética.

- Nanosensores para la detección de contaminantes. La combinación de técnicas de nanomicrofabricación (propias de la microelectrónica) y métodos propios de la química y la biotecnología permiten construir distintos tipos de sensores que pueden tener su aplicación en la detección de contaminantes orgánicos e inorgánicos en el agua.

En la tabla 1 se ilustra la actividad en el campo de la nanotecnología y el agua con ejemplos concretos de investigaciones en marcha o productos ya desarrollados.

Sin embargo, no sólo las nanotecnologías del agua se relacionan con los apartados anteriormente expuestos (que son muy cercanos al tema de la detección y tratamiento de la contaminación del agua). La nanotecnología da mucho más juego. También se puede abordar el tema del agua desde una perspectiva nanotecnológica diseñando nuevos nanomateriales o nanodispositivos para fabricar sensores de diverso tipo ${ }^{13}$ (Cuartero Zueco et al., s/f), realizando estudios sobre el comportamiento de nanofluidos (Carrasco et al., 2009; Karl Johnson et al., 2006), aproximarse al estudio de las propiedades del agua mediante microscopios de sonda local (Gil et al., 2000), sintetizando nanocompuestos que permitan aumentar la solubilidad de compuestos orgánicos en agua, ${ }^{14}$ estudiando sistemas superabsorbentes de agua u otros líquidos (Zhang et al., 2007), obteniendo nuevos polímeros basados en moléculas como las ciclodextrinas capaces de efectuar tareas de descontaminación, ${ }^{15}$ efectuando simulaciones

\footnotetext{
${ }^{13}$ Ver los desarrollos hechos por la empresa española SENSIA (http://www.sensia.es/). En la actualidad SENSIA forma parte del Grupo GENETRIX (http://www.genetrix.es/).

${ }^{14}$ Neowater ${ }^{\mathrm{TM}}$ ( http://www.docoop.com/).

${ }^{15}$ Proyecto "Nanoencapsulación de Derivados del Dibenzofurano mediante Polímeros Hidrosolubles de Ciclodextrina. Aplicación a su Extracción y Preconcentración”, dirigido por Gloria Tardajos Rodríguez y financiado por el Ministerio de Educación y Ciencia.
} 
Mundo Nano | Artículos | Vol. 1, No. 2, Octubre de 2009 | www.mundonano.unam.mx

TABLA 1*

\begin{tabular}{|c|c|c|c|}
\hline $\begin{array}{l}\text { Institución / } \\
\text { Organismo / Empresa }\end{array}$ & País & Descripción de la tecnología & Página web \\
\hline \multicolumn{4}{|c|}{ Tecnologías de nanofiltración por membranas } \\
\hline $\begin{array}{l}\text { Renseelaer Polytechnic } \\
\text { Institute }\end{array}$ & EUA & $\begin{array}{l}\text { Filtros basados en nanotubos de car- } \\
\text { bono para eliminar del agua con- } \\
\text { taminantes de tamaño micro y } \\
\text { nanométrico. }\end{array}$ & http://www.rpi.edu/ \\
\hline $\begin{array}{l}\text { Seldon Laboratorios } \\
\text { (Vermont) }\end{array}$ & EUA & $\begin{array}{l}\text { Filtros basados en nanotubos de car- } \\
\text { bono para eliminar del agua con- } \\
\text { taminantes de tamaño micro y } \\
\text { nanometrico. (Nanomesh (c). }\end{array}$ & $\begin{array}{l}\text { http://www.seldontechnologies. } \\
\text { com/ }\end{array}$ \\
\hline $\begin{array}{l}\text { Banaras Hindu } \\
\text { University }\end{array}$ & India & $\begin{array}{l}\text { Filtros basados en nanotubos de car- } \\
\text { bono para eliminar del agua con- } \\
\text { taminantes de tamaño micro y } \\
\text { nanometrico. }\end{array}$ & http://www.bhu.ac.in/ \\
\hline Argonide & EUA & $\begin{array}{l}\text { Desarrolla filtros basados en nanofi- } \\
\text { bras de alumina depositados sobre } \\
\text { un sustrato de fibra de vidrio (Nano- } \\
\text { ceram()) }\end{array}$ & http://www.argonide.com/ \\
\hline SolmeteX & EUA & $\begin{array}{l}\text { Desarrolla y fabrica membranas ba- } \\
\text { sadas en resinas capaces de capturar } \\
\text { metales pesados y complejos metáli- } \\
\text { cos (arsénicos, mercurio, cadmio, cia- } \\
\text { nida, etc.). }\end{array}$ & http://www.solmetex.com/ \\
\hline Filmtec Corporation & EUA & $\begin{array}{l}\text { Tecnologías de filtración mediante } \\
\text { nanomembranas. }\end{array}$ & $\begin{array}{l}\text { http://www.dow.com/liquidseps/ } \\
\text { inidex.htm }\end{array}$ \\
\hline KX Industries & EUA & $\begin{array}{l}\text { Tecnologías de filtración mediante } \\
\text { nanomembranas fabricadas de po- } \\
\text { límeros, resinas, celulosa, cerámi- } \\
\text { ca, etc. }\end{array}$ & $\begin{array}{l}\text { http://www.kxindustries.com/ } \\
\text { corporate/corp_home.asp }\end{array}$ \\
\hline $\begin{array}{l}\text { North West Univerity, } \\
\text { Potcherstroom }\end{array}$ & $\begin{array}{l}\text { Rep. de } \\
\text { Sudáfrica }\end{array}$ & $\begin{array}{l}\text { Tecnologías de filtración mediante } \\
\text { nanomembranas. }\end{array}$ & $\begin{array}{l}\text { http://www.puk.ac.za/fakulteite/ } \\
\text { natuur/scb/index_e.html }\end{array}$ \\
\hline $\begin{array}{l}\text { University of } \\
\text { Stellenbosch, Institute } \\
\text { of Polymer Science }\end{array}$ & $\begin{array}{l}\text { Rep. de } \\
\text { Sudáfrica }\end{array}$ & $\begin{array}{l}\text { Tecnologías de filtración mediante } \\
\text { nanomembranas. }\end{array}$ & $\begin{array}{l}\text { http://academic.sun.ac.za/ } \\
\text { polymer/ }\end{array}$ \\
\hline FluXXion & Holanda & $\begin{array}{l}\text { Nanomembranas para filtración en } \\
\text { procesos industriales. }\end{array}$ & http://www.fluxxion.com/ \\
\hline Berghof & Alemania & $\begin{array}{l}\text { Nanomembranas para filtración en } \\
\text { procesos industriales. }\end{array}$ & $\begin{array}{l}\text { http://www.berghof-gruppe.de/ } \\
\text { ZUNDEL_HOLDING.html }\end{array}$ \\
\hline Saehan & $\begin{array}{l}\text { Corea del } \\
\text { Sur }\end{array}$ & $\begin{array}{l}\text { Nanomembranas para filtración en } \\
\text { procesos industriales. }\end{array}$ & http://www.saehan.com/ \\
\hline \multicolumn{4}{|c|}{ Zeolitas, arcillas de atapulgita, y polímeros nanoporosos } \\
\hline $\begin{array}{l}\text { Los Alamos National } \\
\text { Laboratory }\end{array}$ & EUA & $\begin{array}{l}\text { Desarrolla nuevos tipos de materiales } \\
\text { poliméricos nanoporosos capaces de } \\
\text { reducir la concentración de contami- } \\
\text { nantes orgánicos comunes del agua } \\
\text { a niveles de una parte por billón. }\end{array}$ & http://www.lanl.gov/ \\
\hline MetaMateria Partners & EUA & $\begin{array}{l}\text { Desarrolla cerámicas nanoporosas } \\
\text { para ser usadas en tecnologías de fil- } \\
\text { tración. }\end{array}$ & http://www.metamateria.com/ \\
\hline
\end{tabular}




\begin{tabular}{|c|c|c|c|}
\hline $\begin{array}{l}\text { Institución / } \\
\text { Organismo / Empresa }\end{array}$ & País & Descripción de la tecnología & Página web \\
\hline $\begin{array}{l}\text { Pacific Northwest } \\
\text { Nacional Laboratory } \\
\text { (PNNL) }\end{array}$ & EUA & $\begin{array}{l}\text { Desarrolla cerámicas nanoporosas so- } \\
\text { bre las que se depositan monocapas } \\
\text { de diversas moléculas para eliminar } \\
\text { contaminantes de forma selectiva. }\end{array}$ & http://www.pnl.gov/ \\
\hline Nanovation AG & Alemania & $\begin{array}{l}\text { Desarrolla cerámicas nanoporosas } \\
\text { para ser usadas en tecnologías de fil- } \\
\text { tración (Nanopore (C). }\end{array}$ & $\begin{array}{l}\text { http://www.itn-nanovation.com/ } \\
\text { main.asp?page=home }\end{array}$ \\
\hline \multicolumn{4}{|c|}{ Tecnologías de desalación mejoradas con nanotecnologías } \\
\hline $\begin{array}{l}\text { The Stephen and Nancy } \\
\text { Grand Water Research } \\
\text { Institute }\end{array}$ & Israel & $\begin{array}{l}\text { Ósmosis inversa con presión aplicada } \\
\text { en el agua salina forzándola a atrave- } \\
\text { sar una membrana nanoporosa. }\end{array}$ & http://gwri.technion.ac.il/ \\
\hline $\begin{array}{l}\text { Long Beach Water } \\
\text { Department }\end{array}$ & EUA & $\begin{array}{l}\text { Estudios del coste energético de la } \\
\text { desalación del agua usando procesos } \\
\text { de nanofiltración en dos etapas. }\end{array}$ & $\begin{array}{l}\text { http://www.waterindustry.org/ } \\
\text { New\%20Projects/desal-20.htm }\end{array}$ \\
\hline \multicolumn{4}{|c|}{ Uso de nanopartículas para degradación catalítica de aguas contaminadas } \\
\hline Inframat Corporation & EUA & $\begin{array}{l}\text { Desarrollo de materiales compues- } \\
\text { tos de estructuras nanofibrosas que } \\
\text { captura arsénico del agua median- } \\
\text { te la combinación de nanofibras de } \\
\text { óxido de manganeso (proceso de } \\
\text { oxidación) con hidróxido férrico na- } \\
\text { nogranular (proceso de adsorción). }\end{array}$ & http://www.inframat.com/ \\
\hline $\begin{array}{l}\text { Enviromental } \\
\text { Care }\end{array}$ & $\begin{array}{l}\text { Hong Kong } \\
\text { (RPC) }\end{array}$ & $\begin{array}{l}\text { Desarrolla tecnologías de oxidación } \\
\text { nanofotocatalítica para la eliminar } \\
\text { bacterias y contaminantes del agua. }\end{array}$ & $\begin{array}{l}\text { http://www.environmentalcare. } \\
\text { com.hk/ }\end{array}$ \\
\hline $\begin{array}{l}\text { University of Illinois, } \\
\text { University of Pittsburg, } \\
\text { Yeshiv University }\end{array}$ & EUA & $\begin{array}{l}\text { Estudios del uso de nanocatalizado- } \\
\text { res para reducir contaminación debi- } \\
\text { da a contaminantes tipo nitratos. }\end{array}$ & $\begin{array}{l}\text { http://www.illinois.edu/ } \\
\text { http:///www.pitt.edu/ } \\
\text { http://www.yu.edu/ }\end{array}$ \\
\hline Rice University & EUA & $\begin{array}{l}\text { Estudio del uso de nanocatalizadores } \\
\text { para eliminación de tricloroetileno de } \\
\text { las aguas subterráneas. }\end{array}$ & $\begin{array}{l}\text { http://cohesion.rice.edu/ } \\
\text { centersandinst/cben/research. } \\
\text { cfm?doc_id=5099 }\end{array}$ \\
\hline $\begin{array}{l}\text { Oklahoma State } \\
\text { University }\end{array}$ & EUA & $\begin{array}{l}\text { Estudio del uso de nanopartículas de } \\
\text { oxido de zinc para eliminar arsénico } \\
\text { de las aguas subterráneas. }\end{array}$ & http://osu.okstate.edu/ \\
\hline \multicolumn{4}{|c|}{ Nanopartículas magnéticas para depuración y tratamiento de aguas } \\
\hline Rice University & EUA & $\begin{array}{l}\text { Desarrollo de nanocristales de } \\
\text { mangnetita para extracción de } \\
\text { arsénico de aguas subterráneas. }\end{array}$ & $\begin{array}{l}\text { http://cohesion.rice.edu/ } \\
\text { centersandinst/cben/research. } \\
\text { cfm?doc_id=5100 }\end{array}$ \\
\hline \multicolumn{4}{|c|}{ Nanosensores para detección de contaminantes y entes patógenos en agua } \\
\hline BioFinger & Europa & $\begin{array}{l}\text { Desarrollo de herramientas portátiles } \\
\text { de detección. }\end{array}$ & http://www.biofinger.org/ \\
\hline University of Buffalo & EUA & $\begin{array}{l}\text { Desarrollo de equipos de detección } \\
\text { de mano capaces de detectar } \\
\text { toxinas usadas en guerra biológica. }\end{array}$ & http://www.buffalo.edu/ \\
\hline University of Maryland & EUA & $\begin{array}{l}\text { Desarrollo de equipos de detección } \\
\text { y filtrado de entes patógenos } \\
\text { que producen enfermedades } \\
\text { gastrointestinales. }\end{array}$ & http://www.umd.edu/ \\
\hline
\end{tabular}

* Esta tabla es una adaptación de las tablas 3-8 que aparecen en Meridian Institute (2006a) y de los contenidos encontrados en Meridian Institute $(2005,2006$ b) y Salamanca-Buentello et al. (2005). 
numéricas y estudios teóricos de las propiedades y estructura del agua ${ }^{16}$ (Franzese et al., 2003), entre otros. Es decir, el tema del agua se entronca perfectamente con la nanociencia (perspectiva básica) y la nanotecnología (perspectiva aplicada).

En el mundo existen multitud de centros de investigación dedicados a los estudios sobre el agua, pero muy pocos de ellos incluyen el agua entre las líneas prioritarias de investigación. A modo de ejemplo, podemos mencionar que en España existen varios institutos ${ }^{17}$ dedicados al estudio del agua desde muy diversas perspectivas (desalación, bio-remediación, filtrado, transporte, gestión de recursos e infraestructuras, etc.), pero en ellos no se detecta actividad visible en el ámbito de las nanotecnologías. De igual manera, para los grupos e institutos de investigación españoles dedicados a nanociencia, el estudio del agua no ha sido nunca una línea prioritaria.

Sin embargo, ha quedado demostrado que la nanotecnología tiene muchos conocimientos y metodologías que ofrecer para el estudio básico y aplicado del agua. Además, el tema del agua preocupa a la ciudadanía de diversos países y en un futuro probablemente condicionará su desarrollo económico.

En el caso español, aunado a este interés político y social, también existe un tejido empresarial considerable relacionado con las tecnologías del agua. Aunque muchas de esta empresas están orientadas a la gestión del agua y a la mera obra civil, hay otras que sí hacen claros esfuerzos en I+D y ocupan posiciones de liderazgo a nivel mundial, sobre todo en el tema de la desalación por osmosis inversa, filtrado, etc. ${ }^{18}$ Por ejemplo, la empresa española ACCionA Agua ${ }^{19}$ ha sido seleccionada para llevar a cabo el diseño, ingeniería, construcción y puesta en marcha de una planta de desalación de agua de mar en California, que será la mayor de Estados Unidos y la quinta del mundo. Dicha planta producirá más de $200,000 \mathrm{~m}^{3}$ / día de agua potable. La misma empresa ha obtenido, en unión con otras compañías, un contrato para construir otra planta desaladora en Australia, con una capacidad de 300,000 m³/día. Por lo tanto, la investigación en este tema sí que tiene un gran potencial de aplicación, dando lugar a una rápida transferencia del conocimiento generado.

Parece claro que hay que aprovechar todos los recursos que tengamos a nuestro alcance para resolver los problemas que la gestión y el tratamiento del agua plantean. La nanotecnología tiene algo que decir al respecto. Países como Israel ${ }^{20}$ o Australia ${ }^{21}$ ya han impulsado la conexión entre "nano" y agua, dado que consideran el tema de los recursos hídricos realmente como prioritario a todos los niveles, incluido el de la I+D. Por lo tanto, siguiendo el ejemplo de estos países es necesario que en algunos de

\footnotetext{
${ }^{16}$ En este sitio se encuentra amplia información acerca de muchos estudios efectuados sobre la estructura del agua http://www.lsbu.ac.uk/water/index.html.

${ }^{17}$ Instituto del Agua, de la Universidad de Granada (http://www.ugr.es/ iagua/). Instituto Universitario del Agua y del Medio Ambiente (INUAMA) de la Universidad de Murcia, (http://www.um.es/inuama/presentacion.php). Instituto Tecnológico del Agua (ITA) de la Universidad Politécnica de Valencia y la Generalitat Valenciana (http://www.ita.upv.es/). Instituto Universitario del Agua y de las Ciencias Ambientales de la Universidad de Alicante, IUACA (http://www.ua.es/institutos/iuaca/instituto/index.html). Instituto Madrileño de Estudios Avanzados IMDEA-Agua de la Comunidad Autónoma de Madrid (http://www.imdea.org/).

${ }^{18}$ Asociación Española de Desalación y Reutilización (http://www.aedyr.com/flash/index.htm).

${ }^{19}$ Acciona-Agua (http://www.acciona-agua.es/).

${ }^{20}$ Para conocer la financiación de Israel a proyectos nanotecnológicos relacionados con el agua, ver la página web http://www.nanoisrael.org/nanoil_research.asp .

21 "Advanced membrane technologies for water treatment research cluster" (Australia) (http://www.csiro. $\mathrm{au} /$ resources/pfuu.html).
} 
los centros emergentes dedicados a nanotecnología que se crean en España, México, Argentina, entre otros países, se incluyan líneas dedicadas al estudio del agua desde las diversas perspectivas antes mencionadas. Incluso se puede pensar en la posibilidad de crear institutos multidisciplinares de investigación dedicados a nanotecnología y al agua.

En conclusión, este campo parece un lugar de encuentro entre el mundo académico y empresarial. Dado que los países deben focalizar sus esfuerzos en I+D hacia sectores concretos, especializándose para ser competitivos en ciertos nichos de mercado; el sector del agua debe, sin duda alguna, convertirse en un referente de los sistemas nacionales de I+D. El desarrollo de tecnologías relacionadas con el agua no sólo permitirá a los países que las generen resolver problemas de tipo doméstico y asegurar su propio abastecimiento, sino que servirá para controlar un conocimiento que permitirá mejorar las condiciones de vida (con beneficios seguros) de varios miles de millones de sedientos habitantes de este planeta. Esperemos que esta oportunidad no se nos escurra entre los dedos.

\section{REFERENCIAS}

Al Gore. (2007). Una verdad incómoda: la crisis planetaria del calentamiento global y como afrontarla. Gedisa, Barcelona. (2007).

Asociación Española de Desalación y Reutilización (http://www.aedyr.com/flash/index. htm).

Carrasco, C., M. Douas, R. Miranda, M. Castellanos, P. A. Serena, J. L. Carrascosa, M. G. Mateu, M. I. Marqués y P. J. de Pablo. (2009). "The capillarity of nanometric water menisci confined inside closed-geometry viral cages". PNAS 106, 5475.

Chapagain, A.K., A. Hoekstra, y H.H.G. Savenije, (2005). "Saving water through global trade", Values of Water Research Report Series, núm. 17. unEsco-IHE, Delft, The Netherlands. Accesible en http://www.waterfootprint.org/Reports/Report17.pdf.

Cuartero Zueco, J., E. Ruiz-Hitzky, P. Aranda y M. Darder. (s/f). Proyecto “Control inteligente de sensores para regular soluciones nutritivas recirculadas y minimizar los vertidos de agua y nutrientes (IFAPA-2002.000890)", financiado por la Junta de Andalucía.

Franzese, G., M. I. Marqués, y H. E. Stanley. (2003). "Intramolecular coupling as a mechanism for a liquid-liquid phase transition”, Phys. Rev. E 67, 011103.

García-Sánchez, A., A. Moyano y P. Mayorga. (2005). "High arsenic contents in groundwater of central Spain", Environmental Geology 47(6) 847.

Gil, A., J. Colchero, M. Luna, J. Gómez-Herrero y A.M. Baró. (2000). “Adsorption of water on solid surfaces studied by scanning force microscopy". Langmuir 16, 5086-5092. A. Gil, J. Colchero, J. Gómez-Herrero y A.M. Baró. (2001) "Different stages of water adsorption on Au by dynamic SFM and jumping mode". Applied Physics A 72, S137S140.

Hoekstra, A.Y. y P.Q. Hung. (2002). Virtual water trade - A quantification of virtual water flows between nations in relation to international crop trade. (UNESCO-IHE, Delft, The Netherlands. Accesible en http://www.waterfootprint.org/Reports/Report11.pdf.

IPCC, The Intergovernmental Panel on Climate Change. (2007). "Climate change 2007. Climate changes impact, adaptation and vulnerability." El informe se puede descargar de la dirección http://ipcc-wg1.ucar.edu/wg1/wg1-report.html. 
Jain, Prashant y T. Pradeep. (2005). "Potential of silver nanoparticle-coated polyurethane foam as an antibacterial water. Filter". Biotech. and Bioengineering 90, 59.

Johnson, J. Karl, John T. Yates Jr. y Oleg Byl. (2006). "Unusual hydrogen bonding in water-filled carbon nanotubes". Journal of the American Chemical Society: JACS, 128, 12090.

Martín-Gil, J., J.M. San-Martín-Toro y J. M. Martín-Villota. (2002). "Problemática planteada por la presencia de niveles elevados de arsénico en el acuífero de los Arenales (sur del Duero)". Tecnoambiente, año XII (118).

Meridian Institute. (2005). "Nanotechnology and the poor: Opportunities and risk". Documento accesible en http://www.meridian-nano.org/gdnp/paper.php.

. (2006a). "Nanotechnology, water, and development". Documento accesible en http:// www.merid.org/nano/waterpaper/.

. (2006b). "Overview and comparison of conventional and nano-based water treatment technologies". Documento accesible en http://www.merid.org/nano/watertechpaper/.

Millenium Project, Task Force on Science, Technology and Innovation. (2005a). Investing in development: A practical plan to achieve the millennium development goals", Earthscan, Londres. El documento es accesible en http://www.unmillenniumproject.org/.

Millenium Project, Task Force on Science, Technology and Innovation. (2005b). Innovation: applying knowledge in development", Earthscan, Londres. El documento es accesible en http://www.unmillenniumproject.org/.

Millenium Project, Task Force on Water and Sanitation. (2005c). Health, dignity, and development: what will it take?. Earthscan, Londres. El documento es accesible en http:// www.unmillenniumproject.org/.

Ng, H.Y. y S.L. Ong. (2006). "Membrane technology for environmental applications-opportunities and challenges", Water Environ. Res. 78, 779-80.

Salamanca-Buentello, F., D.L. Persad, E.B. Court, D.K. Martin, A.S. Daar, and P.A. Singer. (2005). "Nanotechnoogy and the developing world". PLoS Medicine 2, 300-303.

Saxl, Ottilia. (2005). "Nanotechnology - a key technology for the future of Europe". Este documento es accesible en http://www.madrimasd.org/cimtan/Seleccion/.

Schwartz, D. y A. Singh. (1999). Environmental conditions, resources, and conflicts: An introductory overview and data collection. United Nations Environment Programme (UNEP) (ed.). El informe se puede descargar de http://na.unep.net/publications/ Conflicts.pdf.

Smith, Allan H., Elena O. Lingas, y Mahfuzar Rahman. (2000). "Contamination of drinkingwater by arsenic in Bangladesh: A public health emergency", Bull World Health Organ vol. 78, núm. 9. Ginebra.

Srivastava, A., O. N. Srivastava, S. Talapatra, R. Vajtai y P. M. Ajayan. (2004). “Carbon nanotube filtres". Nature Mat. 3, 610-614.

Tiwari, D.K., J. Behari y Prasenjit Sen. (2008). "Application of Nanoparticles in Waste Water Treatment". World Applied Sciences Journal 3, 417.

UNDP (United Nations Development Programme). (2006). "Human Development Report 2006. Power, poverty and the global water crisis”. Palgrave MacMillan, Nueva York. El informe se puede descargar de la dirección http://hdrundp.org/hdr2006/.

UNTWO. (2008). "UNTwo world tourism barometer". UNTWO, vol. 5, núm. 2, junio accesible en http://www.unwto.org/facts/eng/barometer.htm 
Watkins, Kevin y Anders Berntell. (2006). "A global problem: How to avoid war over water", International Herald Tribune, núm. 23, agosto.

WCED, United Nations World Comission on Environment and Development. (1987). Our common future. Oxford, Oxford University Press. Este documento, (también conocido como "Informe Brundtland") es accesible en http://ringofpeace.org/environment/brundtland.html.

Wilf, M. L. Awerbuch, C. Bartels, M. Mickley, G. Pearce y N. Voutchkov. (2007). The guidebook to membrane desalination technology: Reverse osmosis, nanofiltration and hybrid systems process, design, applications and economics". Balaban Pub., Philadelphia.

Zhang, J. H. Chen y A. Wang, (2007). "Study on superabsorbent composites. XV. Effects of ion-exchanged attapulgite on water absorbency of superabsorbent composites", Polymer Composites 28, 208 - 213. 